\title{
Recurrent Ataxia and Dystonia with Anti-Neurochondrin Autoantibodies
}

\author{
Christophe Barrea1이 Frederique Depierreux ${ }^{2}$
} ${ }^{1}$ Department of Pediatrics, Centre Hospitalier Universitaire,
CHU, Liège, Belgium
2Department of Neurology, Centre Hospitalier Universitaire,
CHU, Liège, Belgium

Neuropediatrics

\begin{abstract}
Address for correspondence Dr. Barrea Christophe, MD, Centre Hospitalier Universitaire, Rue de Gaillarmont 600, 4030 Liège, Belgium (e-mail: christophe.barrea@chuliege.be).
\end{abstract}

We report the case of a 7-year-old boy who developed severe recurrent episodes of ataxia. Following the persistence of intrathecal pleocytosis and oligoclonal bands, autoimmune workup was performed, and anti-neurochondrin ${ }^{1}$ antibodies in the cerebro-spinal fluid were discovered. Other investi- gations returned normal, no oncologic accompaniments were found.

Clinical examination identified cerebellar ataxia, cervical dystonia, choreic movements of the upper limbs, action tremor, opsoclonus, dysarthria, and akathisis ( - video 1). Cognitive

Table 1 Physiotherapy and cognitive assessment: significant motor improvement under corticosteroids (0-8 months), with subsequent stabilization under mycophenolic acid (8-18 months); lesser recovery in cognitive functions, including executive, attentional, and visuomotor functions

\begin{tabular}{|c|c|c|c|c|}
\hline & \multirow[t]{2}{*}{ Tests } & \multirow{2}{*}{$\begin{array}{l}\text { Corticosteroid } \\
\text { T0 }\end{array}$} & \multicolumn{2}{|c|}{ Mycophenolate acid } \\
\hline & & & $\mathrm{T} 1$ (6 mo) & $\mathrm{T} 2$ (18 mo) \\
\hline \multirow{5}{*}{$\begin{array}{l}\text { Physiotherapy } \\
\text { assessment }\end{array}$} & MFM D1: standing and transfers & $61.53 \%$ & $76.92 \%$ & $87.18 \%$ \\
\hline & $\begin{array}{l}\text { MFM D2: axial and proximal } \\
\text { motor skills }\end{array}$ & $77.77 \%$ & $94.44 \%$ & $97.22 \%$ \\
\hline & MFM D3: distal motor skills & $80.95 \%$ & $85.71 \%$ & $90.48 \%$ \\
\hline & MFM total: global motor skills & $71.87 \%$ & $85.42 \%$ & $91.67 \%$ \\
\hline & 6 MWT: walking test & 378 m (accompanied) & $\begin{array}{l}408 \mathrm{~m} \\
\text { (alone) }\end{array}$ & $\begin{array}{l}536 \mathrm{~m} \\
\text { (alone) }\end{array}$ \\
\hline \multirow{4}{*}{$\begin{array}{l}\text { Cognitive } \\
\text { assessment }\end{array}$} & Long-term visual memory (NEPSY) & I & $15 / 32(<\mathrm{P} 5)$ & $24 / 32(P 37)$ \\
\hline & $\begin{array}{l}\text { Attentional functions } \\
\text { Alert (KITAP) } \\
\text { Visual attention (NEPSY) } \\
\text { Divided attention (KITAP) } \\
\text { Distractibility (KITAP) } \\
\text { Auditory vigilance (NEPSY) }\end{array}$ & I & $\begin{array}{l}667(\mathrm{P} 1) \\
6(<\mathrm{P} 5) \\
1,180(\mathrm{P} 4) \\
28(\mathrm{P} 8) \\
7(\mathrm{P} 16)\end{array}$ & $\begin{array}{l}710(<\mathrm{P} 1) \\
8(<\mathrm{P} 5) \\
946(\mathrm{P} 14) \\
35(<\mathrm{P} 1) \\
7(\mathrm{P} 16)\end{array}$ \\
\hline & $\begin{array}{c}\text { Executive function } \\
\text { Flexibility (KITAP) } \\
\text { Trail Making Test }\end{array}$ & l & $\begin{array}{l}11(P 4) \\
267^{\prime \prime}\end{array}$ & $\begin{array}{l}12(\mathrm{P} 1) \\
187^{\prime},\end{array}$ \\
\hline & $\begin{array}{l}\text { Visuo-motor precision } \\
\text { (NEPSY) }\end{array}$ & l & $2(<\mathrm{P} 1)$ & $2(<\mathrm{P} 1)$ \\
\hline
\end{tabular}

Abbreviations: 6 MWT, 6 minutes walking test; KITAP, German test battery of attention performance; MFM, Mesure de la Fonction Motrice (measurement scale of motor function); NEPSY, neuropsychological assessment.

received

April 2, 2020

accepted after revision

April 19, 2020 (c) 2021. Thieme. All rights reserved.

Georg Thieme Verlag KG,

Rüdigerstraße 14,

70469 Stuttgart, Germany
DOI https://doi.org/

10.1055/s-0040-1722675. ISSN 0174-304X. 
affective cerebellar syndrome, including neuropsychological impairment associated with significant emotional lability, was also found ( $\mathbf{- T a b l e} \mathbf{1}$ ).

\section{Video 1}

Walking test showing ataxic gait with deviation, instability especially during the turn around, increase space lift and excessive swinging arms; during the second part, the patient experiences difficulties in fine grasping and intention tremor. Note the presence of cervical dystonia (left laterocollis). Online content including video sequences viewable at: https://www. thieme-connect.com/products/ejournals/html/ 10.1055/s-0040-1722675.

Initial corticosteroid therapy (intravenous methylprednisolone $30 \mathrm{mg} / \mathrm{kg}$ for 3 days) resulted in significant improvement. However, following the third relapse, a long-term corticotherapy with monthly intravenous methylprednisolone $500 \mathrm{mg} / \mathrm{m}^{2}$ was initiated. After 8 months, to avoid side effects, this treatment was replaced by mycophenolic acid. No relapse was observed since then. The child currently keeps slightly ataxic gait as well as significant cognitive impairment.
Autoimmune etiologies of the movement disorders are increasingly recognized, even in children., ${ }^{2,3}$ Unexplained repeated episodes of ataxia of subacute onset may require a workup with comprehensive neural IgG screening, especially since immune therapies seem more effective than what is observed in adults. ${ }^{4}$

\section{Funding}

None.

\section{Conflict of Interest}

None declared.

\section{Acknowledgments}

The authors would like to thank Prof. F. Leypoldt for his help and suggestions regarding the laboratory findings.

\section{References}

1 Miske R, Gross CC, Scharf M, et al. Neurochondrin is a neuronal target antigen in autoimmune cerebellar degeneration. Neurol Neuroimmunol Neuroinflamm 2016;4(01):e307

2 Rommel FR, Miske R, Stöcker W, Arneth B, Neubauer BA, Hahn A. Chorea minor associated with anti-neurochondrin autoantibodies. Neuropediatrics 2017;48(06):482-483

3 Weihua Z, Haitao R, Fang F, Xunzhe Y, Jing W, Hongzhi G. Neurochondrin antibody serum positivity in three cases of autoimmune cerebellar ataxia. Cerebellum 2019;18(06):1137-1142

4 Shelly S, Kryzer TJ, Komorowski L, et al. Neurochondrin neurological autoimmunity. Neurol Neuroimmunol Neuroinflamm 2019;6 (06):e612 